\title{
Template Effect in TiN/AIN Multilayered Coatings from First Principles
}

\author{
Deqiang Yin ${ }^{\mathrm{a}, \mathrm{d}}$, Xianghe Peng ${ }^{\mathrm{b}}$, Yi Qin ${ }^{\mathrm{c}, *}$, Zhongchang Wang ${ }^{\mathrm{d}, *}$ \\ ${ }^{\mathrm{a}}$ School of Manufacturing Science and Engineering, Sichuan University, Chengdu, \\ China \\ ${ }^{\mathrm{b}}$ Department of Engineering Mechanics, College of Aerospace Engineering, \\ Chongqing University, Chongqing 400044, China \\ ${ }^{\mathrm{c} D e p a r t m e n t ~ o f ~ D e s i g n, ~ M a n u f a c t u r e, ~ a n d ~ E n g i n e e r i n g ~ M a n a g e m e n t, ~ U n i v e r s i t y ~ o f ~}$ \\ Strathclyde, Glasgow G1 1XQ, Scotland, UK \\ ${ }^{\mathrm{d}}$ Advanced Institute for Materials Research, Tohoku University, 2-1-1 Katahira, \\ Aoba-ku, Sendai 980-8577, Japan
}

\begin{abstract}
Multilayered TiN/AlN coatings find many technological applications where superhardness is suspected to be affected by AlN structures and template effect. Here, we demonstrate, by first-principles calculations on alternative adsorptions of $\mathrm{Al}$ and $\mathrm{N}$ atoms on Ti- and $\mathrm{N}$-terminated TiN surfaces, that the preferred stacking sequences (i.e., having the largest adsorption energy) transform from $f c c$ - to $h c p$ - mode in first a few AlN layers. Using several analytic methods, we identify that for the T-terminated surface, the third added $\mathrm{N}$ layer is critical to inducing the structural transition of AlN, weakening the interaction between the second added $\mathrm{Al}$ and first added $\mathrm{N}$ atoms. The findings provide insight to the complicated template effects in TiN/AlN multilayered coatings, which are practically relevant for further improving property of multilayered coatings at the atomic scale.
\end{abstract}


Keywords: TiN/AlN multilayered coatings; Template effect, First-principles

\section{Introduction}

Multilayered coatings of transition metal nitrides exhibit many unusual physical and mechanical properties [1,2], which render them applicable for many uses, e.g. as engineering tools, magnetic and electric components, and superconducting devices [3]. In particular, multilayered TiN/VN superlattices show a hardness as high as $56 \mathrm{GPa}$, much higher than either of its host coatings [4,5]. To date, there are a few mechanisms for strength enhancement, which are classified as coherency stresses (or supermodulus effects) and nanostructure effect [6]. Chu and Barnett [7] proposed that the difference in shear moduli between the two components may give rise to a high intrinsic stress in coatings, which blocks dislocation motion and leads to a high hardness. On the other hand, Mendibide et al. $[8,9]$ attributed the improved wear resistance of the $\mathrm{CrN} / \mathrm{TiN}$ nanolayers to hindrance of crack propagation. Moreover, metastable phases can form between adjacent layers in multilayered coatings because of the template effect, e.g. in TiN/AIN [10-13]. The high-pressure rocksalt structure of AlN can be stabilized in the epitaxial TiN/AlN superlattices when the layer thickness of AlN is no more than $2 \mathrm{~nm}$. However, a stable hexagonal phase of AlN is formed when the layer thickness is over $2 \mathrm{~nm}$, resulting in a critical thickness of 2 3 $\mathrm{nm}$ for cubic AlN [14-16]. Such template effect is also affected by the orientation of the multilayered coatings, and the preferred orientation of the starting layer determines the preferred orientation of entire coatings [17,18]. For example, the 
orientation in TiN/CrN multilayered coatings shifts from (111) to (200), resulting in significant enhancement of hardness[17].

However, it remains unclear why strength is enhanced in multilayered coatings. Dong et al. [19] synthesized $\mathrm{ZrN} / \mathrm{Si}_{3} \mathrm{~N}_{4}$ nano-multilayers and found that amorphous $\mathrm{Si}_{3} \mathrm{~N}_{4}$ layer is crystallized once its thickness is less than $0.9 \mathrm{~nm}$ owing to the template effect. Wen et al. $[10,20]$ deposited NbN/AlN nano-multilayer films and found that when the AlN thickness is small, both the hexagonal wurtzite-AlN(0002) and face-centered cubic (fcc) $\mathrm{AlN}(111)$ are coherent with fcc $\mathrm{NbN}$ (111), yet further increase of the AlN thickness promotes coherent growth of fcc-NbN(111)/w-AlN(0002). The high hardness of the coatings can be mainly attributed to Koehler mechanism and structural barrier (fcc/hexagonal) to the dislocation motion between $\mathrm{NbN}$ and AlN layers. Such phenomena have also been found in many other metastable nitrides, e.g. TiN/BN [21], CrN/AlN [22,23], $\mathrm{CrN}_{0.6} / \mathrm{TiN}[18]$, and VC/AlN [24,25].

Theoretically, Ivashchenko and Vepřek [26,27] conducted first-principles molecular dynamics calculations of stability and transformations of heterostructures comprising fcc-TiN(001) slab. And, Chawla et al. [28,29] combined the finite element (ABAQUS) and $a b$ initio methods to predict the equilibrium critical thickness up to which the metastable cubic AlN phase is energetically favoured to a stable wurtzite variant in TiN/AlN and CrN/AlN bilayer systems. Chen et al. [30] studied the pseudomorphic growth and the thickness-dependent structural transformation of cubic AlN grown on TiN. Stampfl and Freeman [24] identified initial growth stages and 
compared the critical thickness of TiN/AlN with VN/AlN multilayered coatings. S. Patel et al [31] performed finite element modeling on the structure-property relationship of AlN-TiN composite and estimated the thermal conductivity and thermal expansion coefficient. However, Zhang et al. [32] studied the intrinsic mechanical properties of two AlN polymorphs and found that in spite of its higher elastic modulus, fcc-AlN shows slightly lower shear strength as compared to the stable hcp-AlN. The higher hardness of fcc-TiN/fcc-AlN heterostructures than fcc-AlN/hcp-AlN nanolaminates is attributed not to the difference in intrinsic strength and bonding nature between fcc-AlN and hcp-AlN, yet to formation of semi-coherent interfaces . In view of the general interest of transformation from fcc- to hcp-AlN and intricate growth mechanism in multilayered coatings, we conduct first-principles calculations on growth of AlN on TiN to investigate template effect in the TiN/AlN multilayered coatings.

\section{Calculational details}

Calculations were carried out using Cambridge Sequential Total Energy Package (CASTEP) within the framework of DFT [33]. The pseudopotential was applied for describing the electron-ion interaction, and the generalized gradient approximation of Perdew and Wang (PW91) was used to address exchange-correlation functional [34]. The single-particle Kohn-Sham wave functions were expanded using plane wave with a cutoff energy of $600 \mathrm{eV}$. A vacuum of $30 \AA$ a was embedded into surface supercells to avoid unwanted interactions between slab and its periodic images. Sampling of the 
irreducible edge of Brillouin zone was conducted using the regular Monkhorst-Pack grid with $12 \times 12 \times 12 k$ points for bulk TiN and AlN, and $8 \times 8 \times 1$ for surface and adsorption process $[35,36]$. A series of independent convergence tests were performed, verifying that the above parameters were sufficient to converge total energy to within $0.01 \mathrm{eV} /$ atom.

For $<111>$ TiN surface, a 13-layer slab was found to be thick enough to exhibit bulklike interiors [6]. Six terminations were considered which are divided into two groups in light of termination: $\mathrm{N}$ - and Ti- terminated surfaces with three stacking sequences for each group (Fig. 1). Along the $<111>$ orientation, the atoms stack as the mode of A-B-C-A-B-C--- (hereafter, mentioned as $f c c$-mode), and the stacking sequence of A-B-A-B-- is named as hcp-mode. For adsorption process, an isolated $\mathrm{Al}$ or $\mathrm{N}$ atom was introduced and therefore one stacking sequence was needed to be constructed for each group (Fig. 2). Three stacking sequences were taken into account by placing the added atom ( $\mathrm{Al}$ or $\mathrm{N}$ ) in one of three sites at every step. Figure 2 shows the available positions: Al/N along Ti-N bond (FL: first layer), $\mathrm{Al} / \mathrm{N}$ above $\mathrm{N} / \mathrm{Ti}$ atoms (SL: second layer), and Al/N above Ti/N (TL: third layer). To identify the most energetically stable system at each adsorption step, we calculated adsorption energy $\left(W_{a d s}\right)$, a key quantity in predicting adhesive strength between the substrate and added atom, from $[37,38]$

$$
W_{a d s} \equiv E_{T i N}+E_{M}-E_{T i N / M}
$$

where $E_{T i N}, E_{M}$, and $E_{T i N / M}$ are total energy of isolated TiN slab, $M$ atom $(M=\mathrm{Al}$ or $\mathrm{N})$, and the whole system, respectively. 


\section{Results and discussion}

\subsection{Bulk properties and model geometries}

There are three crystal structures for AlN: rocksalt $(F m-3 m)$, wurtzite $(P 63 M C)$, and zinc-blende $(F 4-3 m)$. The optimum lattice constant of the rocksalt, $h c p$-blend, and zinc-blend AlN are calculated to be $4.07 \AA$, $3.13 \AA(c / a=1.6)$ and $4.39 \AA$, respectively, consistent with the reported values (Table 1). The energy gap of the wurtzite-AlN is calculated to be $4.13 \mathrm{eV}$, in agreement with the reported theoretical values of 4.64 and $4.3 \mathrm{eV}[48,49]$.

The $\mathrm{Al}$ or $\mathrm{N}$ atoms are added alternatively to the Ti- and $\mathrm{N}$-terminated surfaces. All atoms are allowed to relax at every step. To ensure a continuous process of growth, the starting atomic positions at each adsorption step are taken from previously relaxed structure, which has the largest adsorption energy and is viewed as the most stable one. Figure 3 shows the adsorption process for $\mathrm{N}$-terminated surface. The Ti and $\mathrm{N}$ atoms in the first two TiN surface layers are labelled as STi and SN, and the added Al and N atoms are labelled as Alm or $\mathrm{Nm}(\mathrm{m}=1 \sim 7)$, where $\mathrm{m}$ represents the number of newly added layer. A similar process is applied to the Ti-terminated surface. From Fig. 3, one can see that A11 atoms occupy FL layer and the N2 atom also appears at the extended lattice site. However, when the Al3 atom is introduced, the stacking sequences change utterly from $f c c$-mode to $h c p$-mode. When the $\mathrm{N}$ or $\mathrm{Al}$ atoms are subsequently added, the structures maintain the stacking sequence of $h c p$-mode, which indicates that the cubic-AlN layers can exist within three layers only, (4.38 A). Further examination of structure of the $\mathrm{N} 2$ case shows that the $\mathrm{N} 2$ atom pulls the Al1 atom slightly away from 
the N1 or TiN surface, which should account for the structural transition of AlN. In view of the template effect, the thickness of the AlN sandwiched by TiN is doubled, which is comparable to the experimental critical thickness.

Figure 4 shows the fully optimized geometries for the adsorption process of the Ti-terminated surface. As in the N-terminated case, the N1 and Al2 atoms occupy the most stable sites in form of the $f c c$-mode. During the adsorption of N3 and Al4 atoms, the stacking sequence transforms from the $f c c$ - to $h c p$-mode. However, the AlN layers maintain the A-B sequence after the process. The transformation occurs between the step 3 (N-3 with a AlN thickness of $4.04 \AA$ ) and 4 (Al-4 with a AlN thickness of 5.22 $\AA$ ). The Ti-terminated surface is viewed as a shift of the N-terminated surface toward the TiN by one atomic layer (Fig. 3(a) and 4(b)). We hence conclude that the fcc-AlN can exist only in a few layers. We hereafter analyze the Ti-terminated case only due to the similarity in structure between the $\mathrm{N}$ - and Ti-terminated surfaces.

\subsection{Adsorption energy}

Table 2 lists the adsorption energy of the Ti-terminated surface before and after relaxation. For the N1 atom, the FL site exhibits the largest $W_{a d s}$ of 9.16 and $10.15 \mathrm{eV}$ before and after relaxation. For the Al2 atom, the FL site has the largest $W_{a d s}$ of 3.68 before relaxation, but it occupies the TL site after relaxation, indicating the influence of surface on the adsorption process. For the N3 atom, the FL site shows the largest $W_{a d s}$ before relaxation, implying that the stacking sequence obeys $f c c$-mode. However, the FL and SL sites have a similar relaxed $W_{a d s}(9.23$ and $9.25 \mathrm{eV})$, which indicates a weakening effect of surface. In addition, for the Al4 atom, the SL site has the largest 
$W_{a d s}$ before and after relaxation, and the stacking sequence shifts from the $f c c$-mode to $h c p$-mode. The surface effect is therefore weakened substantially with the increase of film thickness and the N-3 atom is critical to the transformation of AlN polymorphs.

\subsection{Partial density of states}

To gain further insight into electronic states, we present in Fig. 5 density of states (DOS) projected on selected atomic layers of the relaxed Ti-terminated surface during the adsorption process. From the fig.5(a), it could be seen that the SN and STi layers are first two layers in TiN surface, and the N1, Al2, N3, Al4, N5, and Al6 denote the added layers at corresponding step. The states for the SN layers are similar, implying that the added atoms impose an insignificant influence on the TiN layers. The adsorption of Al2 atom extends greatly the width of states located mainly from -7.5 to $0 \mathrm{eV}$ and reduces the magnitude of the DOS for the neighbouring $\mathrm{N} 1$ atom. Consequently, when the N3 atom is added, the N1layer shows similar states as in the N1 case. From partial DOS (PDOS) of all layers, the hybridization between the neighbouring layers takes place not only below $E_{\mathrm{F}}$ but within the energy range of 17.5 to $-12.5 \mathrm{eV}$, regardless of the addition of $\mathrm{N}$ or $\mathrm{Al}$, which indicates the presence of covalency in the TiN/AlN layers. The partial density of states for $\mathrm{Al}$ and $\mathrm{N}$ atoms added at step2 to step4 are also presented in Fig.5(b), where on could see that: 1) Al2 in step2 shares the similar distribution in $s$ and $p$ state with Al4 in step4;2) the total state of $\mathrm{N} 3$ in step3 is mainly from $p$ states; 3) the addition of N3(Step3) atoms prominently narrows the width of states, indicating the weakening interaction between 
$\mathrm{A} 12$ and $\mathrm{N} 1$ at the step3; 4) at the step4, the $p$ state of N3 are shifted a little away from the $E_{F}$, accompanied by the similar shift of $s$ and $p$ states of Al2, giving the evidence of enhanced interactions between these two atoms.

\subsection{Charge density and density difference}

To provide a deeper understanding of the adsorption process, we calculate charge density and its difference for the relaxed atomic configurations from step 1 to 4 (Fig. 6). The (110) plane has been chosen purposely so that the slice passes through $\mathrm{N}, \mathrm{Ti}$, and $\mathrm{Al}$ atoms, allowing us to note clearly the majority of bonding interactions between them. Looking at Fig. 6(a), we note that the added N1 atom gains electrons from its neighbouring Ti, enhancing their interaction. When the Al2 atom is added, it supplies electrons to the $\mathrm{N} 1$ atom, implying a weakened interaction between $\mathrm{N} 1$ and Ti atoms. However, once the N3 atom is grown, the Al2 atom somewhat shifts away from the $\mathrm{N} 1$ atom, giving rise to a similar electron distribution of $\mathrm{N} 1$ in between the $\mathrm{N}-1$ and N-3 cases. This phenomenon agrees with the above partial density of states analysis (Fig. 5). Further adsorption of Al4 atom terminates the transition from the $f c c$-mode to $h c p$-mode in view of the stacking sequences of top site between $\mathrm{Al}$ and $\mathrm{N}$ atoms. We thus conclude again that the N3 atomic layer is critical to the template effect.

\section{Conclusions}

We have performed a first-principles study of the adsorption process of $\mathrm{Al}$ and $\mathrm{N}$ atoms on TiN(111) surface, aiming at probing template effect in a TiN(111)/AlN(111) 
multilayered coating. We consider an alternative adsorption of the $\mathrm{Al}$ and $\mathrm{N}$ atoms at three sites on the $\mathrm{N}$ - and Ti-terminated TiN surfaces, and reveal a transition from $f c c$ to $h c p$ - mode regardless of the termination, indicating that the template effect exists in a few AlN layers of only. Further examination of the adsorption energy at each step reveals that the FL and SL sites of the N3 atom have a similar relaxed adhesion energy, implying that the $h c p$-mode tends to occur. Several analytic methods have been used to analyse the adsorption process. We find that the N3 atom mainly gains electrons at the expense of charge loss on its adjacent $\mathrm{Al} 2$ atom, thereby weakening the interaction between A12 and N1 atoms. A transition occurs once the Al4 atom is added, indicating that the $\mathrm{N} 3$ atomic layer is crucial to the template effect.

\section{Acknowledgements}

This study was supported in part by the National Science Foundation of China (grant no. 11302141, 11332013). D.Y. thanks Specialized Research Fund for Doctoral Program of Higher Education (New Teachers) under grant no. 20130181120118. D.Y. and Y. Q. acknowledge European Commission for financial funding of the research through the EU FP7 M3-2S project and the permission for using the CASTEP code as a registered academic license of CASTEP under the STFC/York/Accelrys agreement (UK). Z.C.W. thanks the financial supports from the Scientific Research (B) (grant no. 15H04114), the Challenging Exploratory Research (grant no. 15K14117), the JSPS and CAS under the Japan-China Scientific Cooperation Program, the Murata Science Foundation, and the Shorai Foundation for Science and Technology. 
*E-mails: qin.yi@ strath.ac.uk (Y.Q.); zcwang@wpi-aimr.tohoku.ac.jp (Z.C.W.)

\section{References}

[1] M. Nordin, M. Herranen, S. Hogmark, Influence of lamellae thickness on the corrosion behaviour of multilayered PVD TiN/CrN coatings, Thin Solid Films 348 (1999) 202-209.

[2] V.A. Lavrenko, A.D. Panasyuk, M.Desmaison-Brut, V.A. Shvets, J. Desmaison, Kinetics and mechanism of electrolytic corrosion of titanium-based ceramics in 3\% NaCl solution, J. European Ceram. Soc. 25 (2005) 1813-1818

[3] P.P. Choi, I. Povstugar, Jae-Pyeong Ahn, Aleksander Kosta, Dierk Raabe, Thermal stability of TiAlN/CrN multilayer coatings studied by atom probe tomography, Ultramicroscopy 111 (2011) 518-523.

[4] X. Chu, M.S.Wong, W.D. Sproul, S.L. Rohde, and S.A. Barnett, J. Vac. Sci. Technol. A 10 (1992) 1604.

[5] X. Chu, S.A. Barnett, M.S. Wong, and W.D. Sproul, Surf. Coat. Technol. 57 (1993) 6A. Madan, I.W.Kim, S.C. Cheng, P. Yashar, V.P. Dravid, and S.A. Barnett, Stabilization of cubic AlN in epitaxial AlN/TiN superlattices, Phys. Rev. Lett. 78 (1997) 1743-1746.

[6] D. Yin, X. Peng, Y. Qin, and Z. Wang, Electronic property and bonding configuration at the $\mathrm{TiN}(111) / \mathrm{VN}(111)$ interface, J. Appl. Phys. 108 (2010) 033714

[7] X. Chu, and S.A. Barnett, Model of superlattice yield stress and hardness enhancements, J. Appl. Phys. 77 (1995) 4403-4411

[8] C. Mendibide, P. Steyer, C. Esnouf, P. Goudeau, D. Thiaudiere, M. Gailhanou, J. Fontaine, X-ray diffraction analysis of the residual stress state in PVD TiN/CrN multilayer coatings deposited on tool steel, Surf. Coatings Technol. 200 (2005) $165-169$. 
[9] C. Mendibide, P. Steyer, J. Fontaine, P. Goudeau, Improvement of the tribological behaviour of PVD nanostratified TiN/CrN coatings-An explanation, Surf. Coatings Technol. 201 (2006) 4119-4124.

[10]M. Wen, H. Huang, K. Zhang, Q. Meng, X. Li, L.W. Kong, C.Q. Hu, W.T. Zheng, The AlN layer thickness dependent coherent epitaxial growth, stress and hardness in NbN/AlN nanostructured multilayer films, Surf. Coatings Technol. 235 (2013) $367-375$.

[11]J.W. Lim, S.H. Lee, J.J. Lee, Preparation and properties of nanoscale multilayered TiN/AlN coatings deposited by plasma enhanced chemical vapor deposition, Surf. Coatings Technol. 169-170 (2003) 460-463.

[12]J.L. Endrino, C. Arhammar, A. Gutierrez, R. Gago, D. Horwat ,L. Soriano, G. Fox- Rabinovich, D. Martin y Marero, J. Guo, J.E. Rubensson, J. Andersson, Spetral evidence of spinodal decomposition, phase transformation and molecular nitrogen formation in supersaturated TiAlN films upon annealing, Acta Mater. 59 (2011) 6287-6296.

[13]I.W. Kim, Quan Li, L.D. Marks, and S.A. Barnett, Critical thickness for transformation of epitaxially stabilized cubic AlN in superlattices, Appl. Phys. Lett. 78 (2001) 892-894.

[14]D.G. Kim, T.Y. Seong, and Y.J. Baik, Effects of annealing on the microstructures and mechanical properties of TiN/AIN nano-mutlilayer films prepared by ion-beam assisted deposition, Surf. Coatings Technol. 153 (2002) 79-83.

[15]F.H. Mei, N. Shao, J.W. Dai, G.Y. Li, Coherent growth and superhardness effect of AlN/TiN nanomultilayers, Mater. Lett. 58 (2004) 3477-3480.

[16]M. Stueber, H. Holleck, H. Leiste, K. Seemann, S. Ulrich, C. Ziebert, Concepts for the design of advanced nanoscale PVD multilayer protective thin films, J. Alloys and Compounds 483 (2009) 321-333.

[17]P. Yashar, X. Chu, S.A. Barnett, J. Rechner, Y.Y. Wang, M.S. Wong, and W.D. Sproul, Stabilization of cubic $\mathrm{CrN}_{0.6}$ in $\mathrm{CrN}_{0.6} / \mathrm{TiN}$ superlattices, Appl. Phys. Lett. 72 (1998) 987-989.

[18]Q. Yang, C. He, L.R. Zhao, J.P. Immarigeon, Preferred orientation and hardness 
enhancement of TiN/CrN superlattice coatings deposited by reactive magnetron sputtering, Scripta Mater. 46 (2002) 293-297.

[19] Y.S. Dong, W.J. Zhao, J.L Yue, and G.Y. Li, Crystallization of $\mathrm{Si}_{3} \mathrm{~N}_{4}$ layers and its influences on the microstructure and mechanical properties of $\mathrm{ZrN} / \mathrm{Si}_{3} \mathrm{~N}_{4}$ nanomultilayers, Appl. Phys. Lett. 89 (2006) 121916.

[20]M. Wen, H. Huang, K. Zhang, Q. Meng, X. Li, X.M. Zhang, L.W. Kong, W.T. Zheng, Effects of modulation periodicity on microstructure, mechanical and tribological properties of NbN/AlN nanostructured multilayer films, Appl. Surf. Sci. 284 (2013) 331-339.

[21]R.F. Zhang, S.H. Sheng, S. Veprek, Stability of Ti-B-N solid solutions and the formation of nc-TiN/a-BN nanocomposites studied by combined ab initio and thermodynamic calculations, Acta Mater. 56 (2008) 4440-4449.

[22]G.S. Kim, S.Y. Lee, J.H. Hahn. S.Y. Lee, Synthesis of CrN/AlN superlattice coatings using closed-field unbalanced magnetron sputtering process, Surf. and Coatings Technol.y 171 (2003) 91-95.

[23]J.K. Park, and Y.J. Baik, The crystalline structure, hardness and thermal stability of AlN/CrN superlattice coating prepared by D.C. magnetron sputtering, Surf. Coatings Technol. 200 (2005) 1519-1523.

[24]C. Stampfl, A.J. Freeman, Structure and stability of transition metal nitride interfaces from first-principles: AlN/VN, AlN/TiN, and VN/TiN, Appl. Surf. Sci. 258 (2012) 5638-5645.

[25]G. Li, Y. Li, and G. Li, Crystallization of amorphous AlN and superhardness effect in VC/AlN nanomultilayers, Thin Solid Films 520 (2012) 2032-2035.

[26] V.I. Ivashchenko and S. Vepřek, First-principles molecular dynamics study of the thermal stability of the BN, AlN, SiC and $\mathrm{SiN}$ interfacial layers in TiN-based heterostructures: comparison with experiments, Thin Solid Films, 545: $391-400(2013)$.

[27]V. Ivashchenko, S. Veprek, A. Pogrebnjak, and B. Postolnyi, First principles 
quantum molecular dynamics study of $\mathrm{Ti}_{\mathrm{x}} \mathrm{Zr}_{1-\mathrm{x}} \mathrm{N} /(111) / \mathrm{SiN}_{\mathrm{y}}$ heterostructures and comparison with experimental results, Sci. Technol. Adv. Mater. 15 (2014) 025007.

[28] V. Chawla, D. Holec, P.H. Mayrhofer, The effect of interlayer composition and thickness on the stabilization of cubic AlN in AlN/Ti-Al-N superlattices, Thin Solid Films 565 (2014) 94-100.

[29] V. Chawla, D. Holec, and P.H. Mayrhofer, Stabilization criteria for cubic AlN in TiN/AlN and CrN/AlN bi-layer systems, J. Phys. D: Appl. Phys. 46 (2013) 045305.

[30]D. Chen, X.L. Ma, Y.M. Wang, Thickness-dependent structural transformation in the AlN film, Acta Mater. 53 (2005) 5223-5227.

[31]S. Patel, R. Vaish, N. Sinha, and C.R. Bowen, Finite element analysis of the microstrcuture of AlN-TiN composites, Strain 50 (2014) 250-261.

[32]R.F. Zhang, S.H. Sheng, and S. Veprek, First principles studies of ideal strength and bonding nature of AlN polymorphs in comparison to TiN, Appl. Phys. Lett. 91 (2007) 031906.

[33]S. J. Clark, M.D. Segall, C.J. Pickard, P.J. Hasnip, M.J. Probert, K. Refson, M.C. Payne, First principles methods using CASTEP, Zeitschrift fuer Kristallographite 220 (2005) 567-570.

[34]J.P. Perdew, and Y. Wang, Accurate and simple analytic representation of the electron-gas correlation energy, Phys. Rev. B 45 (1992) 13244-13249.

[35]H.J. Monkhorst, and James D. Pack, Special points for Brillouin-zone integrations, Phys. Rev. B 13 (1976) 135188-135192.

[36]P. Pulay, Ab initio calculation of force constants and equilibrium geometries in polyatomic molecules I. Theory, Molecular Phys. 17 (1969) 197-204.

[37]L.M. Liu, S.Q. Wang, and H.Q. Ye, First-principles study of the effect of hydrogen on the metal-ceramic interface, J. Phys: Condens. Matter 17 (2005) 
$5335-5348$.

[38]L.M. Liu, S.Q. Wang, and H.Q. Ye, Adhesion of metal-carbide/nitride interfaces: Al/TiC and Al/TiN, J. Phys.: Condens. Matter 15 (2003) 8103-811

[39]A. Siegel, K. Parlinski, and U.D. Wdowik, Ab initio calculation of structural phase transitions in AlN crystal, Phys. Rev. B 74 (2006) 104116.

[40]H.Y. Xiao, X.D. Jiang, G. Duan, F. Gao. X.T. Zu, and W.J. Weber, First-principles calculations of pressure-induced phase transformation in $\mathrm{AlN}$ and GaN, Comput. Mater. Sci. 48 (2010) 768-772.

[41]H. Vollstädt, E. Ito, M. Akaishi, S. Akimoto, and O. Fukunaga, High pressure synthesis of rocksalt type of AlN, Proc. Jpn. Acad. B 66B (1990) 7-9.

[42]X.L. Li, H.A. Ma, G.H. Zuo, W.Q. Liu, Y.J. Zheng, J.G. Li, S.S. Li, and X. Jia, Low-temperature sintering of high-density aluminium nitride ceramics without additives at high pressure, Scripta Mater. 56 (2007) 1015-1018.

[43]Y.C. Cheng, X.L. Wu, J. Zhu, L.L. Xu, S.H. Li, and P.K. Chu, Optical properties of rocksalt and zinc blende AlN phases: First-principles calculations, J. Appl. Phys. 103 (2008) 073707.

[44]J.H. Edgar, Properties of Goup-III nitrides, EMIS Datareview Series, London, IEE, 1994.

[45]I. Petrov, E. Mojab, R.C. Powell, and J.E. Greene, Synthesis of metastable epitaxial zinc-blende-structure AlN by solid-state reaction, Appl. Phys. Lett. 60(20) (1992) 2491-2493.

[46]L.H. Shen, X.F. Li, Y.M. Ma, K.F. Yang, W.W. Lei, Q.L. Cui, and G.T. Zou, Pressure-induced structural transition in AlN nanowires, Appl. Phys. Lett. 89 (2006) 141903.

[47]Y.N. Xu, and W.Y. Ching, Electronic, optical, and structural properties of some wurtzite crystals, Phys. Rev. B 48(7) (1993) 4335-4351.

[48]W.M. Yim, E.J. Stofko, P.J. Zanzucchi, J.I. Pankove, M. Ettenberg, and S.L. Gilbert, Epitaxially grown AlN and its optical band gap, J. Appl. Phys. 44 (1973) 292-296.

[49]F. Litimein, B. Bouhafs, Z. Dridi, and P. Ruterana, The electronic structure of 
wurtzite and zincblende AlN: an ab initio comparative study, New J. Phys. 4 (2002) 64.

[50]P.B. Perry, and R.F. Rutz, The optical absorption edge of singlecrystal AlN prepared by a close spaced vapor process, Appl. Phys. Lett. 33(4) (1978) 319-321. 
Table 1 Lattice parameters of the AlN bulk. The reported experimental and theoretical results are also given. The $c / a$ value is shown in bracket in the wurtzite column.

\begin{tabular}{|c|c|c|c|c|}
\hline & & \multicolumn{3}{|c|}{ AlN structure } \\
\hline & & Wurtzite & Zinc-blende & Rocksalt \\
\hline \multirow{4}{*}{$\mathrm{a}(\AA)$} & This work & $3.13(1.60)$ & 4.39 & 4.07 \\
\hline & Experiment & $\begin{array}{l}3.11(1.60)^{39} \\
3.13(1.60)^{40}\end{array}$ & $4.38^{41}$ & $4.05^{42}$ \\
\hline & Theory & $3.12(1.60)^{43}$ & $4.39^{43}$ & $4.06^{43}$ \\
\hline & 1110 o 1 y & $3.13(1.60)^{44}$ & $4.40^{44}$ & $4.07^{44}$ \\
\hline \multirow{4}{*}{$\begin{array}{c}\text { Energy gap } \\
(\mathrm{eV})\end{array}$} & This work & 4.13 & 3.34 & 4.58 \\
\hline & Experiment & $\begin{array}{c}6.28^{45} ; 6.2^{46} \\
6.2 \pm 0.1^{47}\end{array}$ & & \\
\hline & Theory & $3.97^{44} ; \sim 5.0^{50}$ & $3.97^{44}$ & $4.05^{44}$ \\
\hline & meory & $4.64^{48} ; 4.3^{49}$ & $4.0^{50}$ & $5.0^{50}$ \\
\hline
\end{tabular}


Table 2 Adsorption energy for the $\mathrm{N}$ and $\mathrm{Al}$ atoms adsorbed on the Ti-terminated TiN surface with three different stacking sequences at each step (in $\mathrm{eV}$ ).

\begin{tabular}{|c|c|c|c|c|c|c|c|}
\hline \multicolumn{2}{|c|}{ Ti-terminated } & Unrel. & Relaxed & & & Unrel. & Relaxed \\
\hline $\begin{array}{c}\text { Step1 } \\
\text { N1 }\end{array}$ & $\begin{array}{l}\text { FL } \\
\text { SL } \\
\text { TL }\end{array}$ & $\begin{array}{l}9.16 \\
6.07 \\
8.49\end{array}$ & $\begin{array}{l}10.15 \\
6.14 \\
9.52\end{array}$ & $\begin{array}{r}\text { Step2: } \\
\text { Al2 }\end{array}$ & $\begin{array}{l}\text { FL } \\
\text { SL } \\
\text { TL }\end{array}$ & $\begin{array}{l}3.68 \\
2.98 \\
3.05\end{array}$ & $\begin{array}{l}4.26 \\
4.10 \\
4.39\end{array}$ \\
\hline $\begin{array}{c}\text { Step3 } \\
\text { N3 }\end{array}$ & $\begin{array}{l}\text { FL } \\
\text { SL } \\
\text { TL }\end{array}$ & $\begin{array}{l}7.62 \\
7.30 \\
5.36\end{array}$ & $\begin{array}{l}9.23 \\
9.25 \\
5.40\end{array}$ & $\begin{array}{c}\text { Step4 } \\
\text { Al4 }\end{array}$ & $\begin{array}{l}\text { FL } \\
\text { SL } \\
\text { TL }\end{array}$ & $\begin{array}{l}2.80 \\
3.09 \\
2.76\end{array}$ & $\begin{array}{l}4.74 \\
4.81 \\
2.78\end{array}$ \\
\hline $\begin{array}{c}\text { Step5 } \\
\text { N5 }\end{array}$ & $\begin{array}{l}\text { FL } \\
\text { SL } \\
\text { TL }\end{array}$ & $\begin{array}{l}8.77 \\
4.03 \\
8.76\end{array}$ & $\begin{array}{l}9.02 \\
9.21 \\
9.11\end{array}$ & $\begin{array}{c}\text { Step6 } \\
\text { Al6 }\end{array}$ & $\begin{array}{l}\text { FL } \\
\text { SL } \\
\text { TL }\end{array}$ & $\begin{array}{l}2.759 \\
2.741 \\
2.865\end{array}$ & $\begin{array}{l}5.823 \\
5.822 \\
5.827\end{array}$ \\
\hline $\begin{array}{c}\text { Step7 } \\
\text { N7 }\end{array}$ & $\begin{array}{l}\text { FL } \\
\text { SL } \\
\text { TL }\end{array}$ & $\begin{array}{l}4.47 \\
8.04 \\
7.01\end{array}$ & $\begin{array}{l}9.142 \\
9.141 \\
9.217\end{array}$ & $\begin{array}{c}\text { Step8 } \\
\text { Al8 }\end{array}$ & $\begin{array}{l}\text { FL } \\
\text { SL } \\
\text { TL }\end{array}$ & $\begin{array}{l}3.902 \\
4.163 \\
4.864\end{array}$ & $\begin{array}{l}5.437 \\
5.432 \\
5.438\end{array}$ \\
\hline
\end{tabular}


Fig. 1. Schematic plots of TiN(111): (a) N-terminated and (b) Ti-terminated surfaces. The upper part shows top view and low part side view. Only top seven out of thirteen symmetric (111) layers are presented for each termination.

Fig. 2. Schematic plots of (a) OT, (b) SL, and (c) TL stacking sequences. The upper part shows top view and lower part side view. The $\mathrm{N}$-terminated surface and $\mathrm{Al}$ atom are given only (on top of A, B and C).

Fig. 3. Schematic plots for the structure having the largest adsorption energy when the $\mathrm{Al}$ or $\mathrm{N}$ atoms are deposited on the N-terminated TiN surface at each step: (a) Step1, (b) Step2, (c) Step3, (d) Step4, (e) Step5, (f) Step6, and (g) Step7.

Fig. 4. Schematic plots for the structures having the largest adsorption energy when the $\mathrm{Al}$ or $\mathrm{N}$ atoms are deposited on the Ti-terminated TiN surface at each step: (a) Step1, (b) Step2, (c) Step3, (d) Step4, (e) Step5, (f) Step6, (g) Step7, and (h) Step8.

Fig. 5. Density of states projected on selected atomic layers of relaxed Ti-terminated surface during the adsorption process :(a) total density and (b) partial density of states. The Fermi energy is set to zero and denoted by a vertical line.

Fig. 6. Contour plots of charge density for (a) N1, (b) Al2, (c) N3, and (d) Al4, and the corresponding charge density difference for the Ti-terminated surface taken along the 
(110) plane. The charge density difference represents a redistribution of charge in the surface relative to the isolated surface system. The atoms that intersect the contour plane are labeled.

Figure 1
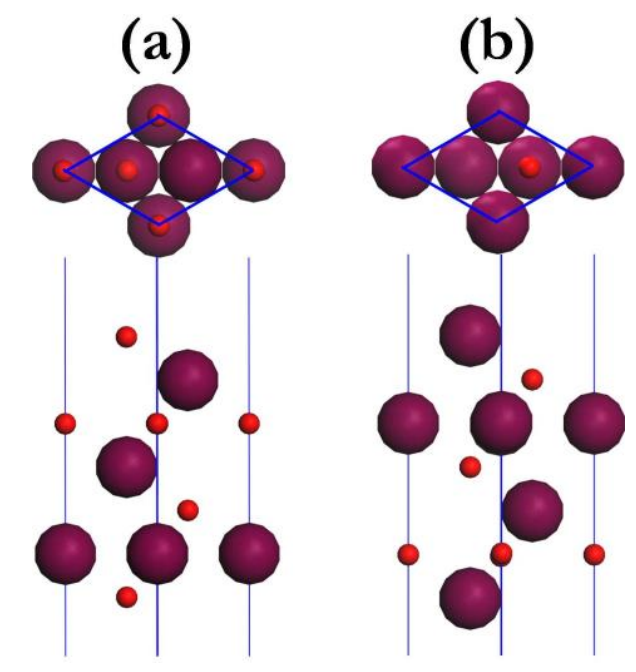
Figure 2

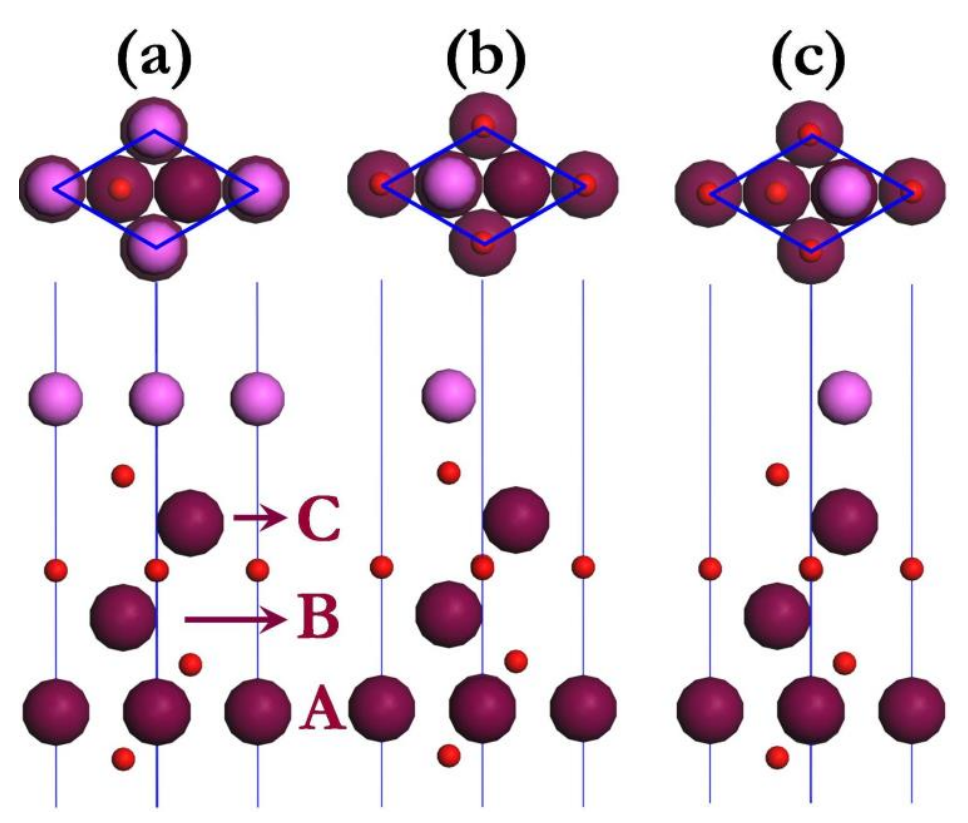

Figure 3

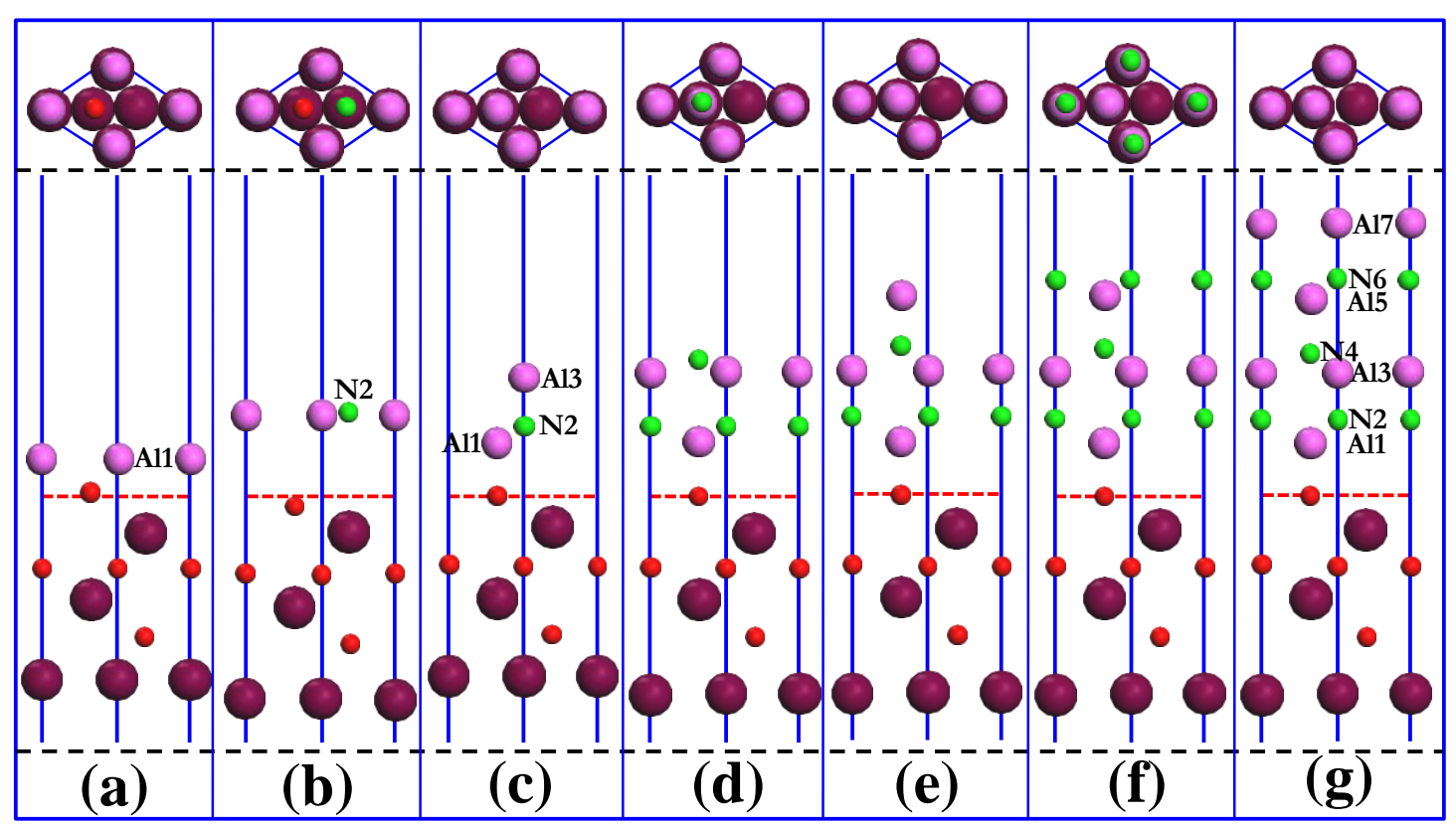


Figure 4

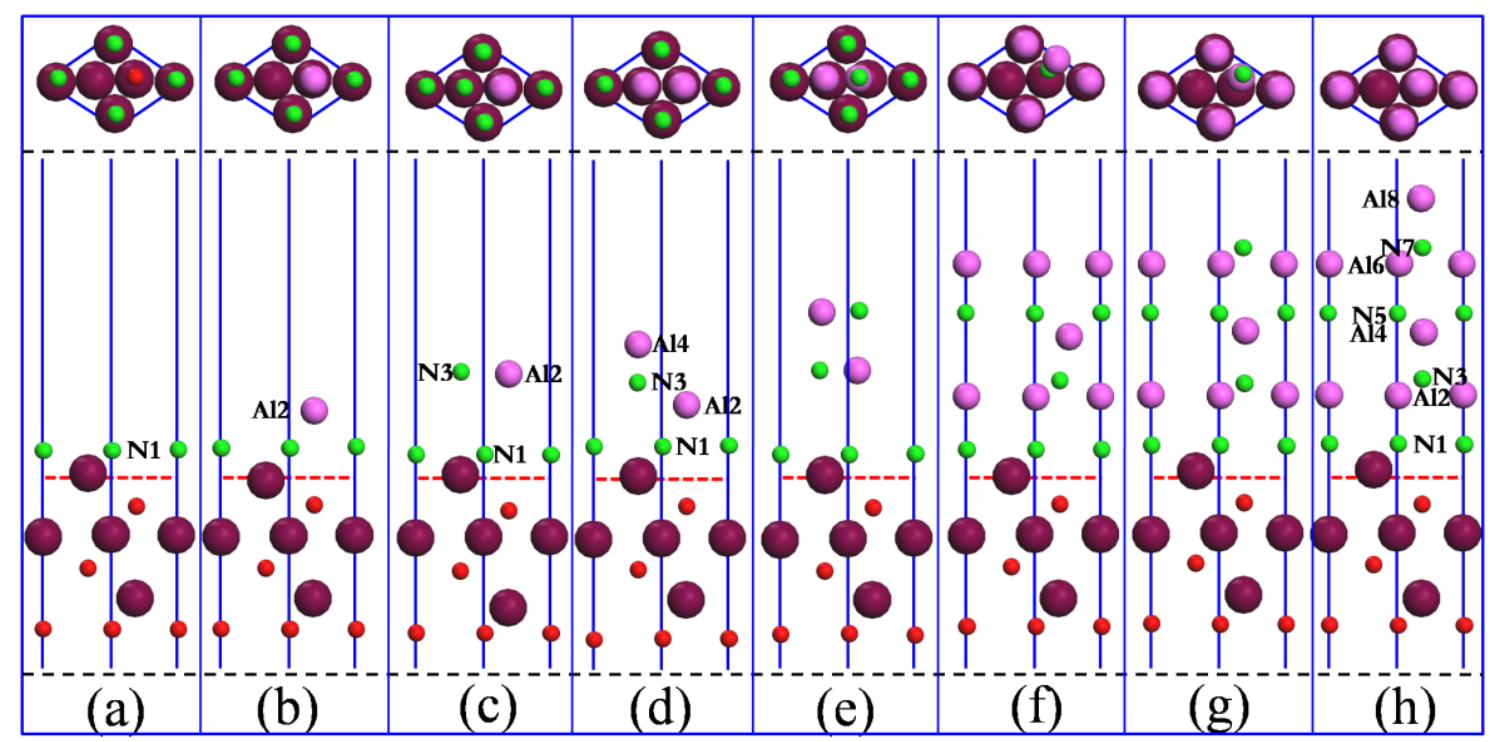

Figure 5

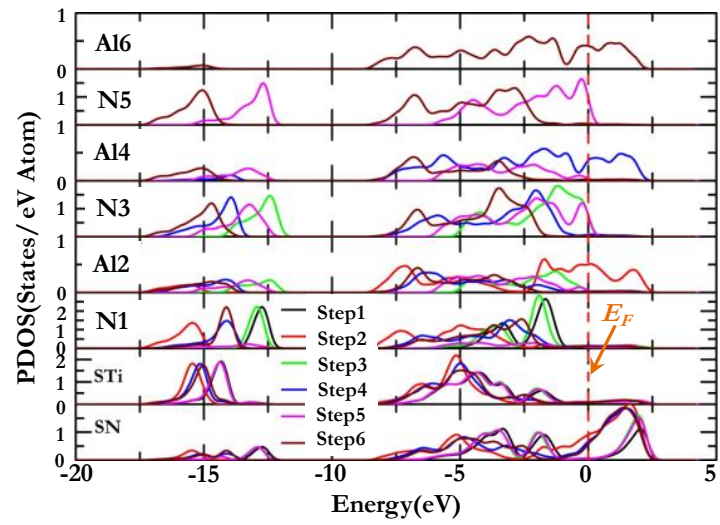

(a)

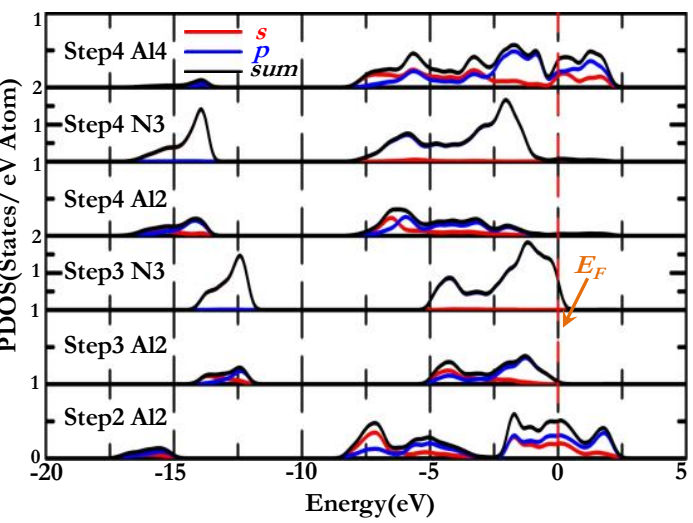

(b) 
Figure 6

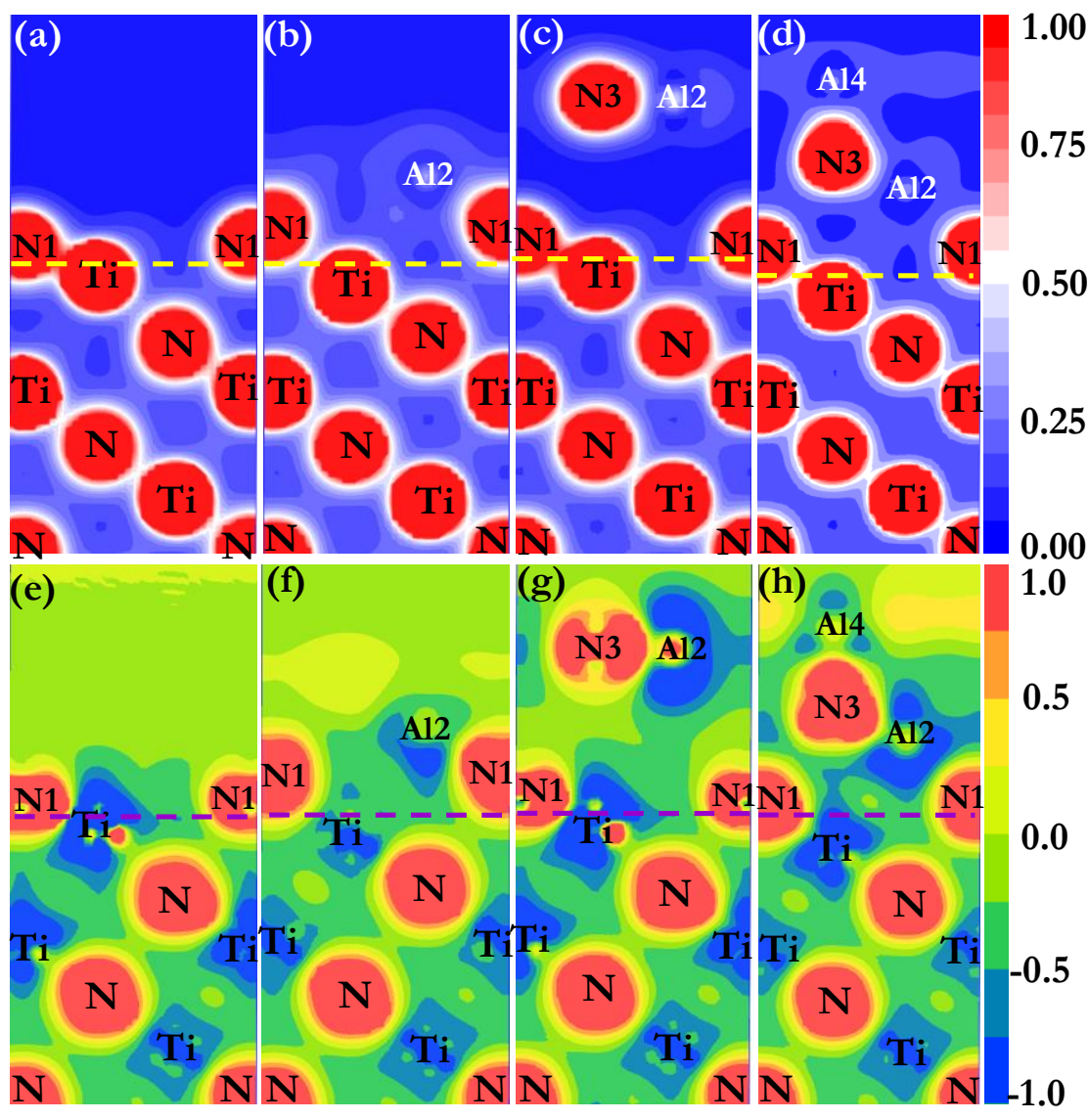

